\title{
Reliability analysis of non-destructive testing models within a probabilistic approach
}

\author{
Wafaa Abdallah ${ }^{1,2, *}$, Jacqueline Saliba ${ }^{2}$, Ziubir-Mehdi Sbartaï $^{2}$, Marwan Sadek ${ }^{1}$, Fadi Hage \\ Chehade $^{1}$, and S.Mohammed ElAchachi ${ }^{2}$ \\ ${ }^{1}$ Université Libanaise, EDST, Hadath, Lebanon \\ ${ }^{2}$ Université de Bordeaux, I2M-GCE, 33405 Talence, France
}

\begin{abstract}
The diagnosis of reinforced concrete is essential to detect the degradation and thus maintain the structural performance of civil engineering structures. This paper aims to establish a mathematical relationship between the ultrasonic pulse velocity UPV (considered as an observable variable) and two concrete properties indicators (compressive strength $f_{c}$ and water content $W$ ) within a probabilistic framework. Synthetic simulations are proposed to derive a conversion model between the statistical properties of the output and the input parameters for a reinforced concrete structure by taking into account spatial variability of concrete.
\end{abstract}

\section{Introduction}

Nowadays, most of the structures for human use (buildings, bridges, etc.) are made of reinforced concrete. Thus, it is of vital importance to ensure that they function correctly during their service life and to detect defect to provide an optimal maintenance strategy[1, $2,3]$. However, the budgets allocated to the maintenance of reinforced concrete structures have reached an alarming level in both developed and developing countries. Therefore, to optimally use these budgets, a rigorous scientific approach is necessary by setting up tools to optimize and make reliable the diagnosis of structure $[1,2,3]$.

The auscultation of reinforced concrete structures means the estimation of the physical or mechanical properties of concrete and their spatial variability. The concrete durability indicators can be assessed by performance-based approaches $[4,5,6]$. The most important indicators are the compressive strength and the water content that reflect the evolution of degradation and forecast the residual service life of reinforced concrete structures $[4,6,7,8]$.

Limitations related to destructive tests sampling, both practical and analytical, leads to the emergence of alternative techniques as non destructive testing (NDT) methods for the auscultation of real structures. NDT techniques are based on well-known physical principles (propagation of mechanical or electromagnetic waves, electric field measurements, etc.). However, many limitations remain with NDT due to the sensitivity of the measured values to multiple parameters, which can lead to major difficulties of

* Corresponding author: wafaa.abdallah@u-bordeaux.fr 
interpretation and high uncertainties when the evaluation has to be quantified [2]. Therefore, robust procedures and inversion techniques must be implemented for data acquisition to evaluate the accuracy of the estimation.

The empirical equations obtained experimentally or found in the literature relating several NDT methods to the mechanical or/and physical properties of concrete are used in this study to evaluate the values measured by NDT based on a probabilistic approach (Monte Carlo numerical simulations). Through these simulations, the necessary parameters characterizing the variability of the ND variables (mean, standard deviation, correlation length, etc.) will be determined. Three technical NDTs (ultrasonic technique, electrical resistivity or Ground Penetrating Radar) are proposed and will be analyzed. The parameters obtained from each NDT method will be then used to evaluate the concrete properties (such as compressive strength). This back analysis allows to find the values of the concrete properties which are now considered as outputs of the numerical simulations. The comparison between the input and output values of these properties allows to compare the experimental results with those obtained by numerical simulations in order to obtain the best numerical modeling and thus a better evaluation of concrete properties.

In this study, synthetic data, derived from the experimental results obtained by [1], are used to perform synthetic simulation. The synthetic data are obtained on a $2 \mathrm{D}$ reinforced concrete slab with a length of $2 \mathrm{~m}$ and a width of $2.5 \mathrm{~m}$. The compressive strength $f_{c}$ and the water content $W$ have been considered as indicators to assess the NDT observables. The ultrasonic pulse velocity $V_{p}$ has been considered in this paper and the "strength-water content- $V_{p}$ “ model has been analyzed as a first step to validate the algorithm of the synthetic simulation.Firstly a section that justify and characterise the spatial variability of concrete has been presented. Secondly, the concrete properties have been combined to evaluate theUPV using numerical simulation. Finally, the methodology of the simulation has been described and validated .

\section{Spatial variability of concrete}

\subsection{Justification}

Determining the spatial variability of concrete properties in reinforced concrete structures can have a great interest for damage assessment or for reliability analyses $[9,10]$. Concrete is a complex and highly heterogeneous material, consisting mainly of three phases: aggregates, cement matrix and a transition zone located at the interface between the cement paste and the aggregates. The spatial variability of concrete results from the variability of the material, as the size, the form or nature of aggregates, its exposure conditions, such as the temperature or humidity, or its studied scale[1,9]. This spatial variability of concrete leads to the use of a probabilistic approach. In the following, the random field theory is presented in order to statistically characterize the spatial variability of a concrete property.

\subsection{Probabilistic Models}

According to [11], the random field (RF) theory should incorporate the observed behavior that: values at adjacent locations are more related than those separated by some distance. For this reason, fundamental statistical properties as the autocorrelation function (ACF) and the variogram have been introduced in addition to the classical statistical parameters, i.e. the mean and the standard deviation (stdv). These two properties make it possible to estimate the degree of correlation between two points by measuring the statistical 
difference between the measured values. They only depend on the distance $h$ between any two distinct points.

In our study, the ACF has been considered as an input for the discretization method of the random field; and the variogram has been chosen to evaluate the correlation length $l_{c}$ of the output parameters after an input RF generation.

It is assumed that the compressive strength $f_{c}$ and the water content $W$ random fields share the same autocorrelation function. An isotropic single exponential autocorrelation function has been used:

$$
\rho\left[(x, y),\left(x^{\prime}, y^{\prime}\right)\right]=\exp \left(-\left(\frac{\left|x-x^{\prime}\right|}{l_{x}}\right)-\left(\frac{\left|y-y^{\prime}\right|}{l_{y}}\right)\right)
$$

Where $l_{x}$ and $l_{y}$ are the autocorrelation distances along $\mathrm{x}$ and $\mathrm{y}$ respectively.

The karhumen-loève methodology presented by [12] has been used in this paper to discretize the random field.

For a series of observed measurements with a limited series of pairs separated by a distance $\mathrm{h}(\mathrm{N}(\mathrm{h}))$, an empirical variogram can be determined as:

$$
\gamma_{e}(h)=\frac{1}{2 N(h)} \sum_{i=1}^{N(h)} \operatorname{Var}\left[Z\left(x_{i}\right)-Z\left(x_{i}+h\right)\right]^{2}
$$

Where $N(h)$ is the number of pairs whose points are separated by a distance $h . Z(x)$ and $\mathrm{Z}(\mathrm{x}+\mathrm{h})$ are two random variables at two points separated by a distance $\mathrm{h}$, denoted $\mathrm{x}$ and $\mathrm{x}+\mathrm{h}$.

For each empirical variogram, a model can be fitted by the least squares method to obtain a mathematical function that can be used later to compute the value of the correlation length $l_{c}$ of the output and the expected value of the measurement at any additional point. It is enough to optimize the value of the parameter $l_{c}$ in the mathematical function obtained by least squares method. The fitting of this variogram is performed with the best theoretical form of variogram (gaussian, exponential, linear,etc ..).

\section{Evaluation of ultrasonic pulse velocity from combining concrete properties}

Ultrasounds are among the most common NDT techniques based on mechanical wave propagation. They are sensitive to mechanical properties such as compressive strength, and also to some physical properties as the saturation rate [1].

To evaluate a NDT, the practice is to use an empirical model linking the properties of concrete to the observed NDT value $\left(V_{p}\left(f_{c}, W\right)\right)$. Thus, concrete properties $\left(f_{c}, W\right)$ are combined here in order to obtain a better assessment of UPVvalues. For the case of $V_{p}$, the experimental data of [1] has been used as input parameters for numerical simulations. The empirical equation obtained by [1] based on experiments has been considered. The shape of this empirical model is:

$$
V_{p}\left(\frac{m}{s}\right)=a * f_{c}(M P a)+b * W(\%)+c
$$

Where the coefficients $a, b$ and $c$ are calculated by minimizing the error between experimental and predicted values according to [1].

\section{Synthetic simulation}




\subsection{Methodology}

The numerical simulations have been performed using MATLAB and Ferum 4.1. The empirical model (equation (3)) makes it possible to give all the input data concerning: the distribution laws of each quantity, their amplitude of variation, as well as their correlation relationships.

A comprehensive step-by-step procedure describing the methodology adopted in the specific case of a spatially varying concrete properties of the slab may be given as follows:

- Define the domain of the slab: in this study, the rectangular slab has dimensions of $2 \mathrm{~m} \times 2.5 \mathrm{~m}$.

- Enter the data of the input random fields: same ACF and autocorrelation length are used for the two RF ( $f_{c}$ and $W$ ). The shape of ACF is exponential as expressed in equation (1) and the autocorrelation length in $\mathrm{x}$ and $\mathrm{y}$ direction is supposed to be $0.5 \mathrm{~m}\left(l_{x}=l_{y}=l\right)$. KL expansion method has been used for the discretization of RF.

- Generate the meshgrid: size of each element is $10 \mathrm{~cm} \times 10 \mathrm{~cm}$.

- This step consists to identify and designate the random fields and to define the marginal distribution for each RF (type, mean, standard deviation (stdv)). The two $\mathrm{RF}\left(f_{c}\right.$ and $\left.W\right)$ are supposed to have a normal distribution: $f_{c} \sim N\left(\mu_{f c}=\right.$ $30 \mathrm{MPa}, \sigma_{f c}=5$ (Coefficient of variation $\left.\operatorname{COV}_{f c}=16.67 \%\right)$ ) and $W \sim N\left(\mu_{W}=6 \%, \sigma_{W}=0.9\left(\operatorname{COV}_{W}=15 \%\right)\right)$.

- Generate randomly the two RF for the total number of simulations (NS).

- Compute the correlation length of $f_{c}$ and $W$ after this generation denoted $l_{c}$ and compare it to that used for the input data $l$. The way to determine the correlation length is based on the variogram. The histogram of the distribution values of $f_{c}$ and $W$ is plotted after the random generation, and fitted with the normal probability density function (PDF). The obtained mean and stdv of each PDF should be compared to those used for input RF. These comparisons are necessary to validate the method of the random generation.

\subsection{Validation}

In order to validate the random generation of each input random field, ten simulations have been calculated to obtain the best input value of the autocorrelation length for both random fields. In the sequel, only five random generations are presented. The best fitting of histograms for values of each input $f_{c}$ and $W$ after the random generation was the normal probability density function. Table (1) shows the characteristics of normal PDF. In comparison with the entrance characteristics of normal PDF of $f_{c}$ and $W\left[f_{c} \sim N\left(\mu_{f c}=\right.\right.$ $\left.30 \mathrm{MPa}, \sigma_{f c}=5\right)$ and $\left.W \sim N\left(\mu_{W}=6 \%, \sigma_{W}=0.9\right)\right]$, table 1 shows for each of the five random generations, accepted values of the mean (in the range of $30 \mathrm{MPa}$ for $f_{c}$ and $6 \%$ for $W$ ), but the values of stdv are slightly different from the entrance values.

Table 1. Characteristics of normal PDF of $f_{c}$ and $W$ of five simulations with $l=0.5 \mathrm{~m}$.

\begin{tabular}{|l|l|l|l|l|l|l|}
\cline { 2 - 7 } \multicolumn{1}{c|}{} & simulation number & 1 & 2 & 3 & 4 & 5 \\
\hline \multirow{3}{*}{$\mu$} & $f_{c}(\mathrm{Mpa})$ & 31.28 & 32.92 & 29.62 & 30.57 & 30 \\
\cline { 2 - 7 } & $W(\%)$ & 6.07 & 6.28 & 6.34 & 5.33 & 6.16 \\
\hline \multirow{3}{*}{$\sigma$} & $f_{c}(\mathrm{Mpa})$ & 1.74 & 2.46 & 2.42 & 3.57 & 2.3 \\
\cline { 2 - 7 } & $W(\%)$ & 0.46 & 0.42 & 0.49 & 0.45 & 0.54 \\
\hline
\end{tabular}


Figure 1 shows the histogram of the input random field $W$ after the random generation (first simulation presented in table 1) and the corresponding fitting using normal probability density function (curved line in this figure).

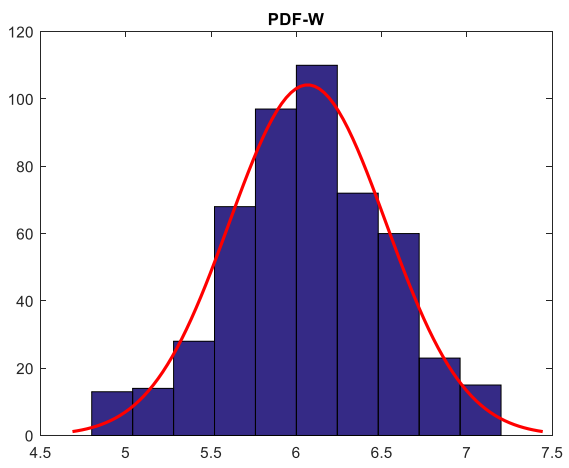

Figure 1. PDF of $W$ for the first random simulation presented in Table 1- case $l=0.5 \mathrm{~m}$

In order to compare the values of the correlation length for the two input random fields before and after the random generation, Table 2 gives the values of correlation lengths of $f_{c}$ and $W$ for five from ten simulations.

Table 2. Correlation length $l_{c}$ for $f_{c}$ and $W$ of five simulations with $l=0.5 \mathrm{~m}$.

\begin{tabular}{|c|c|l|l|l|l|l|}
\hline \multirow{4}{*}{$l_{c}(\mathrm{~m})$} & Simulation number & 1 & 2 & 3 & 4 & 5 \\
\cline { 2 - 7 } & $f_{c}$ & 0.895 & 0.506 & 0.736 & 0.628 & 0.99 \\
\cline { 2 - 7 } & $W$ & 0.448 & 0.672 & 0.558 & 0.582 & 0.657 \\
\hline
\end{tabular}

Also, for an illustration step of the obtained results, Figure 2 shows the corresponding variogram fitting of the input random field $W$ after the random generation for the ten simulations calculated (only five simulations are presented in Table 2). As shown in this figure, the fitting is also based on a Gaussian law. The results show that for each of the five random generations, $l_{c}$ is different from the input value $(l=0.5 \mathrm{~m})$. Thus, a parametric study that takes into account the variation of input autocorrelation length $l$ should be considered.

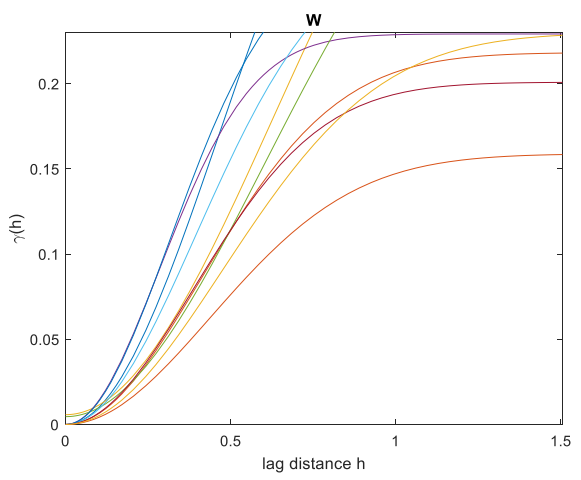

Figure 2. Variogram of $W$ for each random simulation with $l=0.5 \mathrm{~m}$. 


\section{Conclusion}

Ten numerical simulations for a specific case of input data for the two random fields $\left(f_{c}\right.$ and $W$ ) have been calculated to validate the random generation method of the input random fields. The obtained results are promising. In order to improve the time computational efficiency, the same procedure given in paragraph (4.1) will be implemented using another discretization method (e.g. Circulant Embedding CE). Mention the calculation time with the actual method (specification of the PC also). The effect of the spatial variability of the random fields $\left(f_{c}\right.$ and $W$ ) on the UPV and thus the empirical model is currently studied. Finally, an inversion procedure will be developed for concrete properties evaluation using NDT techniques.

\section{References}

1. N. T. Nguyen, Z.M. Sbartaï, J.-F. Lataste, D. Breysse, F. Bos « Assessing the spatial variability of concrete structures using NDT techniques: Laboratory tests and case study ", Const. Build. Mater., 49, p.240-250 (2013).

2. D. Breysse, «Evaluation non destructive en place du béton : un problème usuellement mal posé.», 21st French Congress of Mechanics, (2013).

3. D. Breysse, "Analyse des facteurs conditionnant la qualité de l'évaluation non destructive d'un ouvrage en béton armé : application au cas d'un tablier de pont», XXXIth Meeting AUGC-IBPSA Cachan, (2013).

4. G. Villain, V. Garnier, Z.M. Sbartaï, X. Dérobert, J.-P. Balayssac, « Development of a calibration methodology to improve the on-site non-destructive evaluation of concrete durability indicators», Mater. Struct., 51, 40 (2018).

5. M.G. Alexander, Y. Ballim, K. Stanish «A framework for use of durability indexes in performance-based design and specifications for reinforced concrete structures », Mater. Struct. , 41, p. 921-936 (2008).

6. V. Baroghel-Bouny, «Concrete design for a given structure service life-durability management with regard to reinforcement corrosion and alkali-silica reaction-state of the art and guide for the implementation of a predictive performance approach based upon durability indicators ", Scientific and technical documents AFGC, (2007).

7. A. Raharinaivo, G. Arliguie, T. Chaussadent, G. Grimaldi ,V. Pollet, G. Tache, « La corrosion et la protection des aciers dans le béton », Pres. Eco. natio. Pon. Chaus., (1998).

8. A. Bentur, S. Diamond, N.S. Berke, «Steel corrosion in concrete-fundamentals and civil engineering practice », E\&FN SPON, London, (1997)

9. C.Gomez-Cardenas, Z.M. Sbartaï, J.-P. Balayssac, V. Garnier, D. Breysse «New optimisation algorithm for optimal spatial sampling during non-destructive testing of concrete structures, Eng. Struct., 88, p.92-99 (2015).

10. J.W. Van Groeningen, W. Siderius, A. Stein, «Constrained optimization of soil sampling for minimization of the kriging variance », Geoderma, 87, p. 239-259 (1998).

11. E.H. Vanmarcke, "Random fields: Analysis and synthesis.", M.I.T Press, Cambridge, Mass., 382 p, (1983).

12. P.D. Spanos, R. Ghanem, "Stochastic finite element expansion for random media." Jour. Eng. Mec., ASCE, 115, 5 (1989). 